Recherches en histoire de l'art, histoire des civilisations, archéologie, anthropologie et muséologie

$16 \mid 2021$

Cahier $n^{\circ} 16$

\title{
La Jeune Grecque de David d'Angers ou le rêve brisé
}

Greece Revived by David d'Angers or the broken dream

\section{Philippe Durey}

\section{OpenEdition \\ Journals}

Édition électronique

URL : https://journals.openedition.org/cel/13033

DOI : $10.4000 /$ cel. 13033

ISSN : 2262-208X

Éditeur

École du Louvre

\section{Référence électronique}

Philippe Durey, "La Jeune Grecque de David d'Angers ou le rêve brisé », Les Cahiers de l'École du Louvre [En ligne], 16 | 2021, mis en ligne le 21 mai 2021, consulté le 30 juillet 2021. URL : http:// journals.openedition.org/cel/13033; DOI : https://doi.org/10.4000/cel.13033

Ce document a été généré automatiquement le 30 juillet 2021.

\section{(c) (i) (9)}

Les Cahiers de l'École du Louvre sont mis à disposition selon les termes de la licence Creative Commons Attribution - Pas d'Utilisation Commerciale - Pas de Modification 4.0 International. 


\title{
La Jeune Grecque de David d'Angers ou le rêve brisé
}

\author{
Greece Revived by David d'Angers or the broken dream
}

Philippe Durey

1 En Grèce, à Missolonghi, dans le Jardin des Héros, sont réunis les monuments dédiés aux combattants des quatre sièges successifs subis par la petite ville pendant la guerre d'indépendance (1821-1829). Se détachant dans une nature verdoyante, placée sur un socle assez élevé en forme de tombeau porté par un emmarchement, on peut y voir la figure rêveuse d'une très jeune fille nue, coiffée d'un élégant chignon (fig. 1), dont la présence peut sembler étrange en un tel lieu. Avec l'index de sa main droite, elle épelle les lettres d'un nom gravé sur la dalle ornée d'une croix, tandis que de sa main gauche, sur laquelle elle s'appuie, elle tient une couronne de lauriers. Il s'agit d'une Jeune Grecque déposant une couronne sur le tombeau de Marco Botzaris, mort héroïquement le 21 août 1823 au cours du deuxième siège de la ville, telle que l'a imaginée le sculpteur français Pierre-Jean David d'Angers (1788-1856), qui en a fait don à la Grèce ${ }^{1}$. 
Figure 1.

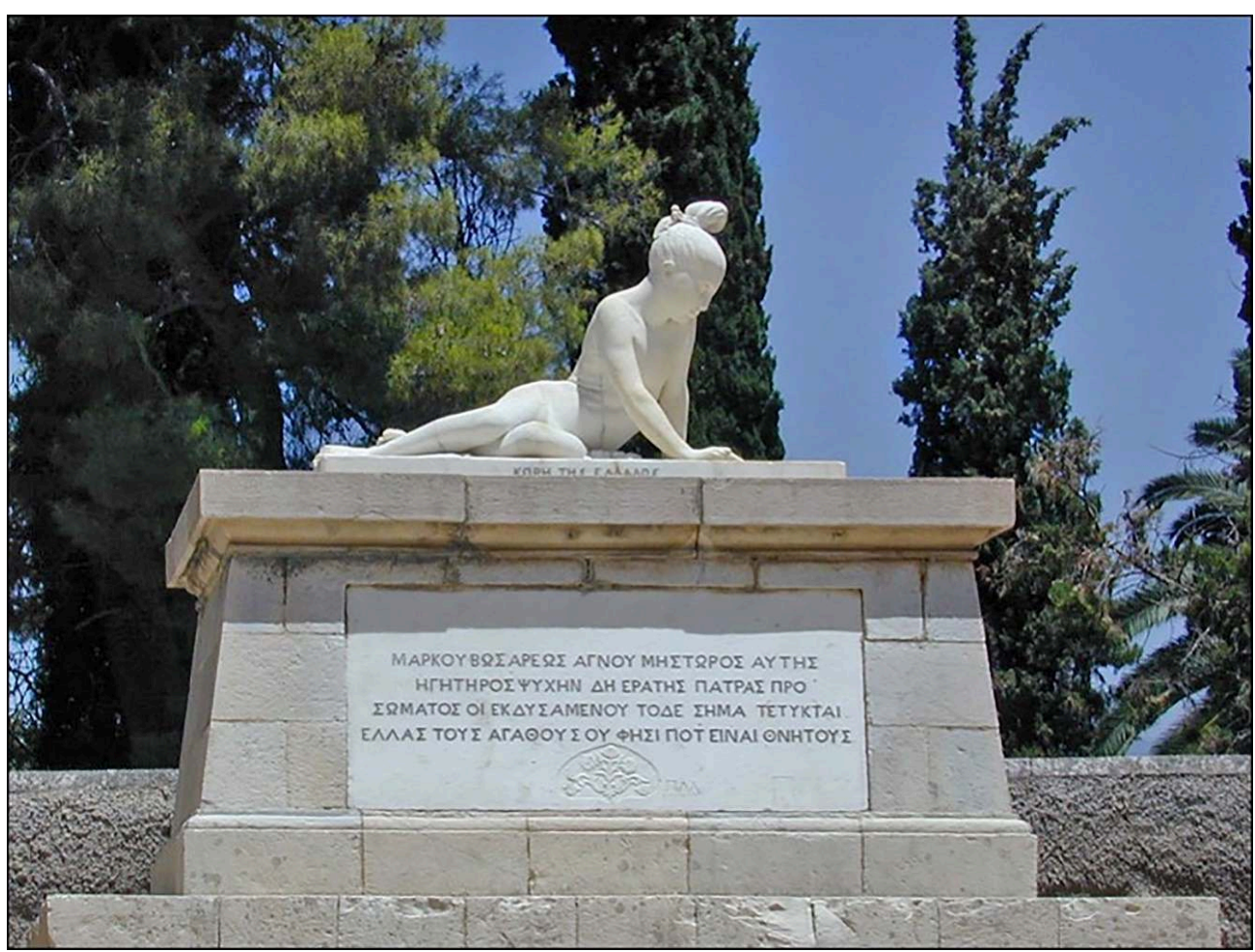

David d'Angers, La Jeune Grecque, Missolonghi, Jardin des Héros.

(c) Jean-Pierre Dalbéra

2 Bien que son nom figure sur la tranche de la terrasse, le marbre que l'on peut admirer aujourd'hui à Missolonghi n'est qu'une copie de l'œuvre de David; une copie de belle qualité, commandée en 1915 par le Premier ministre Vénizelos à Georgios Bonanos (1863-1940), important sculpteur grec d'inspiration classique ${ }^{2}$. Le marbre original est exposé au musée historique d'Athènes dans un environnement bien différent. Il y est présenté sur un socle bas, devant un fond rouge très muséal, scandé de pilastres solennels, avec la note romantique de quelques boulets de pierre posés sur le sol juste à côté (fig. 2). Ce qui frappe le visiteur, au-delà du naturel et de la délicatesse de cette figure, c'est l'inclusion de marbre plus blanc qui apparaît très visible sur le côté de la tête de la jeune fille, en fait une restauration qui semble avoir mal vieilli (fig. 3). D'autres se laissent voir sur les mains et les pieds. 
Figure 2.

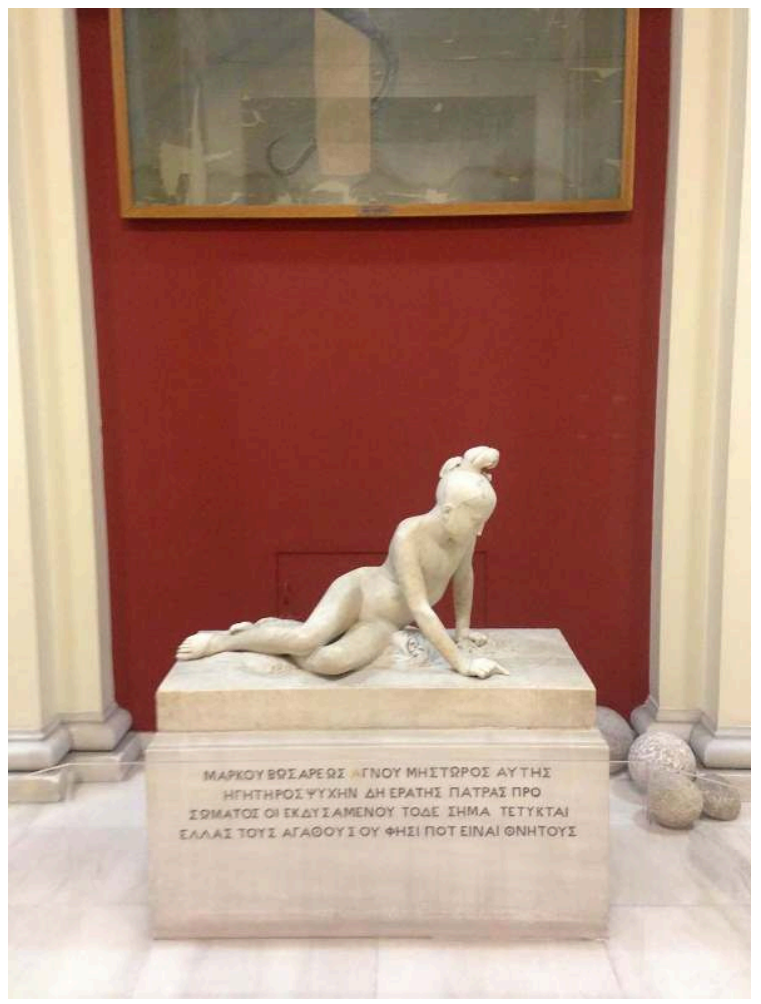

David d'Angers, La Jeune Grecque, Athènes, musée national historique. (C) Philippe Durey 
Figure 3.

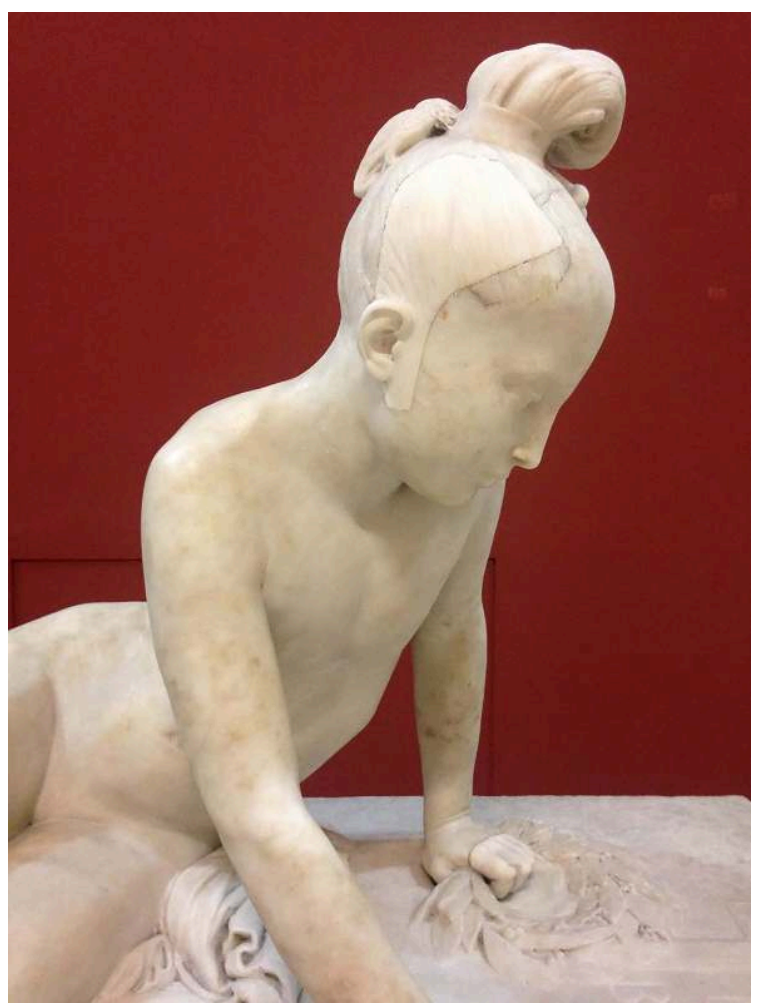

David d'Angers, La Jeune Grecque, détail, Athènes, musée national historique. (c) Philippe Durey

3 L'œuvre est importante, non seulement sur le plan historique, mais aussi sur le plan artistique. Elle occupe dans la sculpture française de la première moitié du XIX ${ }^{e}$ siècle une place comparable à celle du célèbre tableau de Delacroix peint en 1826, représentant $L a$ Grèce expirant sur les ruines de Missolonghi (Bordeaux, musée des BeauxArts). Si elle n'a pas la fougue colorée, ni le caractère puissamment dramatique de la sombre vision du peintre, la jeune fille de David parvient à créer un symbole poétique d'une étonnante présence, une image troublante et mélancolique, opposant la jeunesse et la mort, la chaude souplesse d'un corps d'enfant et la froide rigidité d'une dalle de tombeau... Elle symbolise la jeune Grèce contemporaine, dans sa nudité et pauvreté originelles, méditant le souvenir des héros qui se sont sacrifiés pour elle ${ }^{3}$.

Pourtant, l'œuvre reste bien moins connue en France que le tableau de Delacroix. Il y a à cela plusieurs raisons. La première tient à la place secondaire de la sculpture par rapport à la peinture dans la conscience culturelle du public contemporain, surtout pour le XIX siècle, à l'exception sans doute de Rodin. L'autre tient à la perception des créations de David, relativement peu représenté au Louvre comme dans la plupart des grands musées de Beaux-Arts, et connu surtout pour ses médaillons, pour quelques bustes de personnalités célèbres et dans une moindre mesure, pour le fronton du Panthéon. Son œuvre est visible principalement à Angers, dans la belle galerie qui porte son nom, installée depuis 1984 dans les ruines, couvertes d'un toit en verre, d'une ancienne abbaye gothique du XIII ${ }^{\mathrm{e}}$ siècle, l'église Toussaint. Y sont conservés en pleine lumière presque tous les plâtres originaux de David, tous les modèles de ces « colosses radieux ", chantés par Victor Hugo pour évoquer les statues des grands hommes dont il a parsemé la France. Parmi eux, le plâtre modèle de La Jeune Grecque (fig. 4). 
Figure 4.

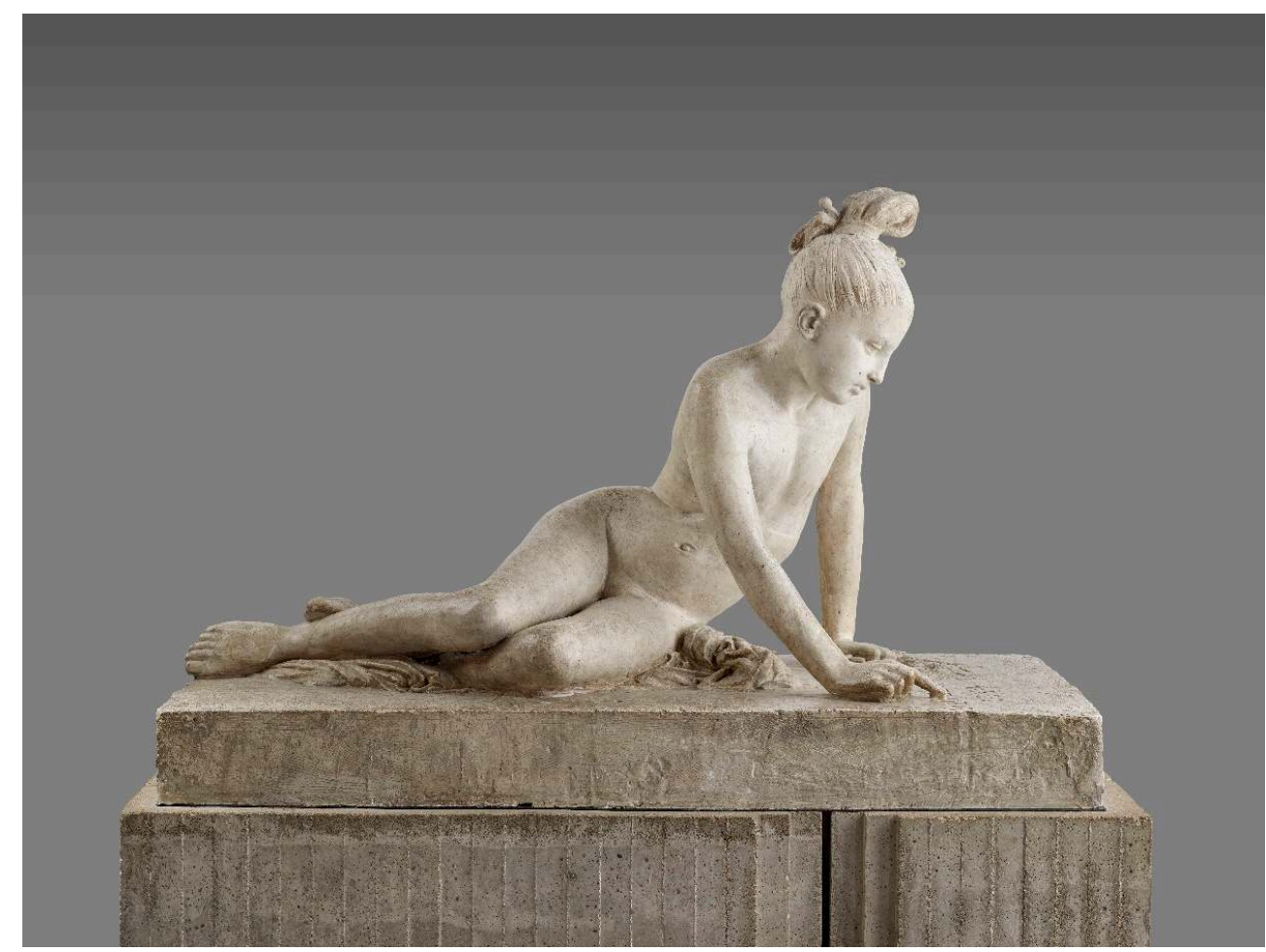

David d'Angers, La Jeune Grecque, Angers, Galerie David d'Angers, inv. MBA 856.15.

(C) RMN-Grand Palais/Benoit Touchard

5 Si l'intérêt de David pour Botzaris et la cause grecque, la façon dont il trouva son idée et son attachement à sa statue sont bien connus, une partie de l'histoire est restée beaucoup plus approximative, parfois même obscure : la date exacte de réalisation de l'œuvre, la connaissance qu'on en eut en France avant son envoi en Grèce, les circonstances de cet envoi, les contacts avec les Grecs, la place de David dans le mouvement philhellène. Encore moins étudiés sont la relation sentimentale et phantasmatique du sculpteur avec la Grèce et ses projets d'y partir installer une école de sculpture ; moins perçus enfin, sa déception brutale, son « rêve brisé » lors de ses retrouvailles avec la statue, en Grèce, en 1852. Le sort de sa Jeune Grecque y tient sa part, mais la déception relevait aussi d'un contexte plus global et de l'état d'esprit dans lequel se trouvait alors le vieux sculpteur.

Cet article entend éclairer les aspects méconnus ou mal connus de cette histoire, non seulement parce qu'elle concerne une œuvre importante d'un grand artiste du $\mathrm{XIX}^{\mathrm{e}}$ siècle et raconte un moment des relations entre deux pays, la France et la Grèce, mais aussi parce que le récit de cette transplantation ratée - peut-être impossible d'un artiste déjà consacré est révélateur du cheminement culturel difficile, long et douloureux, d'une jeune nation dans le concert européen de l'époque. 


\section{L'histoire d'une œuvre}

\section{La naissance du projet}

7 Le texte suivant est bien connu et souvent reproduit, mais il est nécessaire de repartir de lui pour comprendre l'histoire de l'œuvre. Notamment le délai, pas moins de onze années, qui va s'écouler entre la mort de Marco Botzaris le 21 août 1823 et le départ du marbre de David pour la Grèce à l'automne 1834.

" Aussitôt que j'eus connaissance de la mort de Marco Botzaris, je formai le projet de lui élever un monument. Longtemps je cherchai dans mes souvenirs allégoriques une pensée qui put rendre dignement ma profonde admiration pour ce grand homme, mais tout me paraissait emphatique. J'attendis l'inspiration. Un jour, me promenant dans un cimetière, je vis une petite fille, à genoux sur un tombeau, épeler avec son doigt l'inscription qui y était gravée. J'avais trouvé ma composition ».

8 Ces lignes écrites en 1834, dans un texte intitulé Une nuit d'atelier, ont été retrouvées et publiées par le grand biographe du sculpteur, Henry Jouin, dans l'ouvrage fondamental qu'il lui a consacré en $1878^{4}$. Si elles indiquent comment David a trouvé l'idée de sa composition, elles ne disent malheureusement pas à quel moment et précisent simplement que David chercha " longtemps » et qu'il « attendit l'inspiration ». Pour en savoir un peu plus, on dispose heureusement du témoignage d'un contemporain célèbre, Victor Hugo, rapporté par son épouse Adèle :

«Un jour que M. Victor Hugo allait chez la mère Saguet avec M. David, ils rencontrèrent, rue du Montparnasse, une fille de treize à quatorze ans en guenilles ; M. David la regarda, s'arrêta, lui parla, et prit note de son nom et de son adresse. M. Victor Hugo, étant allé voir M. David dans son atelier la semaine suivante y rencontra la pauvre petite, nue, grêle, étiolée, flétrie par la misère, et pourtant belle. M. David en faisait la jeune fille au tombeau de Botzaris, laquelle, dans sa pensée, représentait la Grèce, alors opprimée et souffrante ${ }^{5}$ ».

Or, on peut dater cette scène, sans grand risque d'erreur, de 1827. C'est en effet l'année au cours de laquelle David fait la connaissance de Victor Hugo, par l'intermédiaire de Louis Pavie ${ }^{6}$, et noue avec lui une amitié durable, faite d'admiration réciproque.

C'est donc en 1827 que David réalise son modèle en terre qu'il fait ensuite mouler pour obtenir le plâtre-modèle conservé aujourd'hui à Angers. Or cette année-là, David a en vue un évènement capital pour tous les artistes du temps, l'exposition du Salon, qui ouvre le 4 novembre. Dans le livret, il fait inscrire, parmi les quinze œuvres qu'il veut y présenter, sous le numéro 1083: «Jeune fille grecque déposant une couronne sur le tombeau de Botzaris. Cette statue est en marbre des Pyrénées ». C'est cette mention qui est à l'origine de l'information que l'on trouvera ensuite partout répétée et qui est pourtant fausse, selon laquelle le marbre aurait été présenté au Salon de 1827.

11 Car il est assez fréquent à l'époque que les artistes soient non seulement en retard pour exposer leurs œuvres dès l'ouverture du Salon, mais n'aient même pas le temps de les $\mathrm{y}$ envoyer avant sa fermeture. Il existe aux Archives des musées nationaux des registres d'entrées dans lesquels l'administration du musée notait l'arrivée des œuvres et parfois la date de cette arrivée. On n'y trouve nulle part mention de La Jeune Grecque ${ }^{7}$. Par ailleurs, les journaux de l'époque multipliaient les commentaires et les critiques : on ne trouve là encore aucune trace de La Jeune Grecque, alors qu'il est peu vraisemblable que celle-ci n'ait suscité aucune réaction. D'autant que c'est au Salon de 1827, qui ouvre 
quelques jours à peine après la bataille de Navarin, que figurent le plus grand nombre d'œuvres évoquant la guerre d'indépendance grecque ${ }^{8}$. David est alors l'un des sculpteurs les plus en vue, en passe d'atteindre vers 1830 le sommet de sa carrière... Si elle avait été exposée, l'œuvre aurait été décrite et analysée. Le 12 mars, le Journal des Débats indique d'ailleurs en page 2: «Le catalogue annonce plusieurs statues de M. David: le Grand Condé, Racine, Talma, etc. Ces ouvrages ne sont point encore exposés [le journal se trompe pour le Racine, exposé depuis le 9 mars ${ }^{9}$ ]. On ne voit de M. David que les portraits [c'est-à-dire les bustes] de Fénelon, de M. Casimir Delavigne, Bentham et d'autres [...] ». Que s'est-il passé ? Pourquoi une telle différence entre ce qui était annoncé et ce qui fut réellement montré?

En fait, le 6 janvier 1828, David fut victime dans la rue d'une très violente agression, reçut une grave blessure à la tête qui faillit lui coûter la vie et qui l'obligea à s'aliter pendant trois mois ${ }^{10}$. Il n'était donc plus en mesure d'achever avant la fin du Salon en avril tout ce qu'il avait prévu d'exposer et notamment pas le marbre de sa Jeune Grecque qu'il avait entrepris - fait exceptionnel sur lequel nous reviendrons - de tailler seul, sans l'aide de ses praticiens. La conclusion s'impose : La Jeune Grecque ne figura ni en marbre, ni même en plâtre au Salon de 1827.

\section{De l'ébauche à l'envoi}

Se pose alors la question de la date de réalisation du marbre ou, plus exactement, celle des étapes de sa réalisation. Car sur le marbre lui-même figure, sur la tranche de la terrasse, une inscription, sans doute postérieure ${ }^{11}$, portant le nom du sculpteur et la date de 1834 (fig. 5). Curieusement, cette date est restée inconnue d'Henry Jouin, alors qu'elle figure aussi sur certaines réductions en bronze éditées par le fondeur Barbedienne à partir de $1855^{12}$. À la suite de Jouin, l'inscription et la date sont restées ignorées des monographies et catalogues consacrés à David et c'est seulement en 1987 qu'une première photographie de l'œuvre est publiée par le conservateur du musée historique d'Athènes, Georges Geroyamis-Petimezas, dans un article édité en France, révélant au public français de nombreux documents d'archives grecs apportant des précisions très intéressantes sur l'histoire de l'œuvre ${ }^{13}$. 
Figure 5.

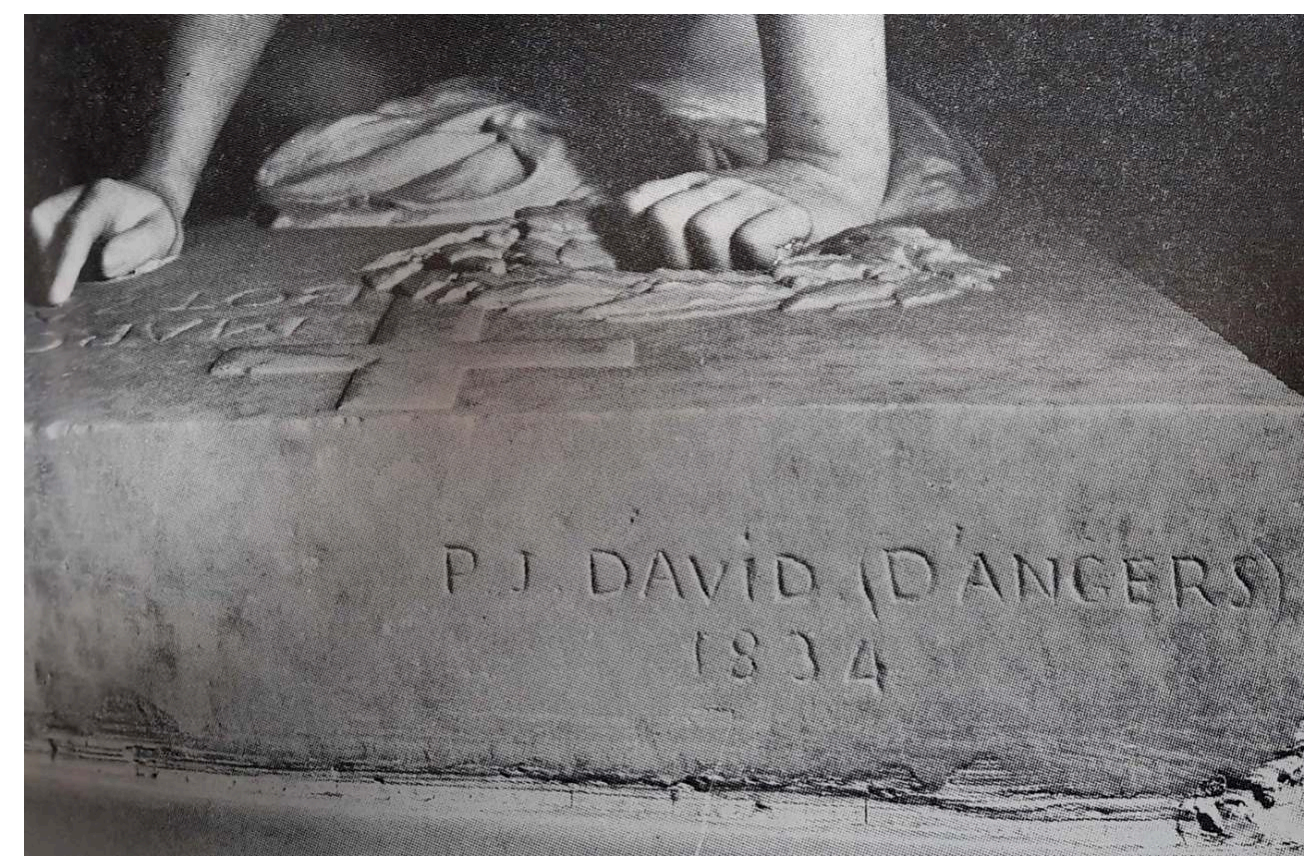

Geroyannis-Petimezas, «La Présence de David d'Angers en Grèce », image IV., Marie-Rose et

Marguerite-Cécile Albrecht, David d'Angers, regards autour d'un sculpteur, Maulévrier, Hérault éditions.

Pourquoi s'écoule-t-il sept ans entre la réalisation du modèle en plâtre en 1827 et l'achèvement du marbre en 1834 ? Il y a plusieurs raisons, mais la principale tient au besoin qu'a le sculpteur de trouver des interlocuteurs pour faire parvenir son marbre à sa destination, c'est-à-dire sur la tombe de Botzaris, et pour faire accepter officiellement son don. Une femme de lettres irlandaise, Lady Sydney Morgan (1776-1859), qui passe dans l'atelier entre avril et juin $1829^{14}$, admire tout particulièrement La Jeune Grecque (sans doute voit-elle, aux côtés du plâtre-modèle, le marbre en cours d'exécution) et indique à ses lecteurs : « c'est un présent de l'auteur au gouvernement grec et il regarde l'acceptation de ce fruit de son talent par un peuple libre comme la plus haute récompense qu'il puisse recevoir $»^{15}$. L'emploi du conditionnel semble bien indiquer que David attend encore à cette date l'acceptation de son don. Or cette validation, il l'attendit, comme nous allons le voir, jusqu'à la fin de 1833.

Par ailleurs, David veut tailler ce marbre seul, sans l'aide de ses praticiens. Sur ce point, on dispose du témoignage catégorique d'Adrien Maillard, l'un de ses amis et son premier biographe, le 21 octobre 1841 :

"Aucun des praticiens, aucun des nombreux élèves du maître n'y a travaillé; aucun. Lui seul l'a, de ses veilles et de ses mains, rêvée, modelée, ciselée, enrichie de tous les détails de la nature et de l'art. Lui seul [...] l'a, durant le silence des nuits, qu'il attendait de préférence, fouillée, caressée du ciseau aux lueurs de la lampe ${ }^{16}$ ».

Ce travail, le soir ou la nuit, à la lumière de la lampe, ne doit pas étonner. Dans ses carnets, David le recommande et il est d'usage fréquent chez les sculpteurs du temps. La lumière rasante de la flamme, que l'on déplace autour du marbre, permet en effet de mieux mettre en relief les subtilités du modelé. Mais, chose plus rare, tel un nouveau Pygmalion, David fait une véritable fixation affective et sentimentale sur sa statue. Voilà comment il lui dit adieu en 1834, dans le texte déjà cité plus haut, Une nuit 
d'atelier: «Te voilà terminée, chère enfant, tu vas quitter notre France pour ce beau pays de Grèce ! Je t'aimais tant ! Ah! Je t'aimais comme un père tendre aime sa fille, même malgré ses défauts qu'il connait si bien !17 ». Quand il la retrouvera en 1852 à Missolonghi, sur le monument de Botzaris, il écrira dans ses Carnets : « De très loin, j'ai aperçu la statue de la jeune fille qui regarde son nom sur la pierre tombale. Il m'a semblé voir ce jeune enfant tressaillir, en voyant son créateur d'il y a trente années! ${ }^{18}$ ». Même en faisant la part du style très romantique des écrits de David, il est certain qu'il a nourri une relation très particulière avec cette œuvre, dont il a sûrement tardé à se séparer et dont il gardera d'ailleurs le plâtre chez lui jusqu'à sa mort ${ }^{19}$.

Compte tenu de ce que nous venons de dire, il est normal que David insiste auprès de certains de ses amis pour qu'ils viennent voir son marbre terminé, quand il l'expose à titre privé pendant quelques jours galerie Vivienne en août 1834 juste avant le départ pour la Grèce. Il invite ainsi personnellement Lamartine ${ }^{20}$, mais aussi Chateaubriand ${ }^{21}$. Des critiques d'art font paraître leurs commentaires dans la presse. Certains posent la question d'une éventuelle référence à un modèle antique (ils ont sans doute en tête la Joueuse d'osselets) mais plutôt pour l'écarter. Ainsi le Journal des Artistes : « M. David n'a sans doute pas voulu faire un antique de cette jeune fille, qu'il a représentée nue au lieu de la draper du vêtement hellénique de l'antiquité et à laquelle il a donné des formes très ordinaires au lieu de se rapprocher des formes typiques de la beauté ». Tout en reconnaissant le profond naturel de la figure, la traduction parfaitement juste de «la maigreur relative des membres, à cet âge de transition où l'enfant n'est pas encore une jeune fille accomplie », le critique reproche à David de lui avoir donné les pieds d'une "gardeuse de vaches", d'en avoir fait une fille du peuple, bref, d'avoir mis du romantisme dans sa figure ${ }^{22}$. On notera cependant qu'en renonçant aussi à tout costume grec contemporain, à tout pittoresque facile ${ }^{23}$, David donne à sa figure un caractère intemporel et en fait un symbole universel, selon les règles de la sculpture classique. D'autres qui apprécient l'œuvre, comme Gustave Planche, ne comprennent pas pourquoi David ne l'a pas exposée au Salon: «M. David se doit à lui-même de montrer [...] les ouvrages dont il décore la France et dont plusieurs iront au-delà des mers pour ne plus nous revenir. Son Jefferson est aujourd'hui à Philadelphie. Encore quelques jours et sa Jeune fille grecque partira par les soins du prince Soutzo ${ }^{24}$ ».

\section{Acceptation officielle de l'hommage par la Grèce}

Ce "prince Soutzo» est Michalis Soutzos (1778 ou 1784-1864), alors ambassadeur de Grèce à Paris. Comment David avait-il donc fait connaître et accepter par les Grecs son projet de don? Indiquons d'abord que La Jeune Grecque faillit devenir un cadeau officiel du gouvernement français à l'État grec. Selon François Grille, homme de lettres angevin qui fut sous l'Empire et la Restauration fonctionnaire au ministère de l'Intérieur, le vicomte de Martignac, chef du gouvernement de Charles X de janvier 1828 à août 1829, en eut l'intention. Il le proposa au roi qui refusa ${ }^{25}$. Sans doute la forme républicaine prise par le gouvernement grec de Kapodistrias déplaisait-elle profondément à Charles X. David mit ensuite du temps pour trouver les bons interlocuteurs. Les troubles politiques en France liés à la Révolution de juillet 1830, puis ceux de Grèce après l'assassinat de Kapodistrias le 9 octobre 1831, ne lui facilitèrent certainement pas la tâche. Une solution ne fut trouvée qu'après l'installation de la monarchie d'Othon I ${ }^{\text {er }}$ en 1833. 
19 C'est un intellectuel grec vivant depuis longtemps en France, Michel Schinas (1792-1870) qui ouvrit à David les contacts officiels nécessaires. Ce phanariote ${ }^{26}$, érudit et polyglotte, avait publié en 1825 un Tableau moral et politique de la Grèce et surtout, en 1829, une Grammaire élémentaire du grec moderne qui eut en France un réel succès. Il fut nommé la même année membre scientifique de l'expédition de Morée, attaché à la section archéologique. Il avait par ailleurs rédigé en 1824 un Éloge funèbre de Botzaris ${ }^{27}$. Est-ce par l'Institut, dont David était membre depuis 1826, et dont dépendait en partie la commission de Morée, que les deux hommes se rencontrèrent? Nous l'ignorons. Quoiqu'il en soit, revenu en Grèce en 1833, Schinas envoie de Nauplie le 6 septembre une note à la Régence :

«Ayant appris que Sa Majesté, lors de son passage à Missolonghi [il s'agit sans doute du voyage d'arrivée d'Othon en Grèce] a daigné faire concevoir de flatteuses espérances, que sous ses auspices, le tombeau de M. B. recevrait une apparence digne des services rendus à la patrie par ce héros, j'ai pensé qu'il ne doit pas lui rester inconnu qu'étant à Paris, M. David, membre de l'Institut (Académie des Beaux-arts) et l'un des artistes les plus distingués de France, m'a fait voir dans son atelier un monument funéraire qu'il préparait, de son propre mouvement, pour la tombe de M. B. ».

Suit une brève description et ce commentaire : «l'expression de sa figure était d'une tendre et naïve tristesse et ce travail entier de la plus grande beauté28 ». Dans les semaines qui suivent, la Régence demande à Alexandros Mavrocordatos (1791-1865), le ministre des Affaires étrangères, de se renseigner et si possible d'obtenir un dessin. La réponse arrive le 22 mars 1834 dans une lettre que Mavrocordatos adresse au roi :

« M. David ayant déclaré à M. Soutzo [...] sa bonne volonté d'offrir cet ouvrage à la Grèce aussitôt qu'il aura achevé d'y mettre la dernière main [on constate ici qu'à cette date David n'a pas encore achevé son marbre] et ayant acquis par là des titres de reconnaissance du Gouvernement Royal, j'ose signaler cet acte à Votre Majesté en lui soumettant respectueusement en même temps mon opinion de lui conférer la croix d'argent de l'ordre du Sauveur ${ }^{29} »$.

21 Le temps ensuite que l'œuvre soit terminée, qu'elle soit exposée en août à la galerie Vivienne, puis envoyée à Marseille pour prendre le bateau pour la Grèce, elle n'arrivera à Nauplie qu'en novembre ${ }^{30}$.

22 Le principe de la décoration proposée par Mavrocordatos avait été accepté dès le 30 mars 1834 par le roi, mais sans être suivi d'effet. Le 12 mai 1835, le nouveau ministre des Affaires étrangères, Jacovos Rizos (1778-1850), le rappelle au roi et le décret est pris le 25 juillet $^{31}$. Mais l'envoi de la décoration à David par le nouvel ambassadeur à Paris, Ioannis Kolettis (1774-1847), n'intervient que près d'un an plus tard, le 21 mai 1836 ${ }^{32}$. La presse française s'en fait l'écho le 17 juillet dans le Journal des Beaux-Arts qui enjolive assez largement :

«Le monument funèbre consacré à Botzaris par M. David a été reçu et inauguré à Missolonghi avec une grande reconnaissance pour cet artiste, auquel le roi Othon a fait remettre la croix de l'ordre de la Délivrance [sic] en décidant qu'un jeune grec serait entretenu aux frais de l'État dans l'atelier de M. David pour y étudier la sculpture ${ }^{33} »$.

Or, ces informations sont à la fois approximatives (l'ordre du Sauveur est devenu, sans doute en raison d'une mauvaise traduction, l'ordre de la Délivrance), et fausses: l'inauguration officielle en présence du roi Othon et de la reine Amélie n'aura lieu en effet que deux ans plus tard, le 18 octobre $1838^{34}$. Quant à l'envoi d'un jeune grec aux 
frais de l'État dans l'atelier de David, nous n'en avons pas trouvé d'autre trace, mais nous reviendrons plus loin sur la question de l'atelier.

La Jeune Grecque semble avoir attendu assez longtemps à Missolonghi qu'on la mit en place. Dans une lettre du 6 septembre 1837 adressée au roi ${ }^{35}$, le secrétaire d'État pour l'Intérieur, Anastassios Polyzoïdés (1803-1872), déclare ne pas vouloir "entrer dans l'examen des motifs pour lesquels cette affaire a été retardée sous mes prédécesseurs ». Il préfère attirer l'attention royale sur les questions qui restent à résoudre : d'abord trouver un emplacement pour le monument (cette statue de jeune fille nue ne pouvait évidemment pas être installée dans l'église où était enterré Botzaris) et lui imaginer ensuite un «piédestal» ou un socle pour lequel il propose au roi un dessin... Finalement, comme nous le savons, l'emplacement retenu sera le Jardin des Héros, créé par Kapodistrias.

\section{II - Le philhellénisme de David}

Il faut se pencher maintenant sur les autres aspects du philhellénisme de David, car cela est indispensable si l'on veut comprendre la décision qui le poussera en 1852 à choisir la Grèce comme terre d'asile.

\section{Un philhellénisme actif}

Il y a d'abord sa relation personnelle avec la sculpture antique ; elle remonte bien sûr à ses années de formation à l'École des Beaux-Arts à Paris, à son prix de Rome et aux années passées en Italie. Elle est profondément marquée par la révélation des marbres du Parthénon, qu'il va voir à Londres dès son retour en 1816. Il est d'ailleurs l'un des premiers sculpteurs français à faire cette démarche. La sculpture grecque, mais aussi la cité grecque antique et ses héros, resteront pour lui, toute sa vie, une référence et une source de questionnement.

Il n'a certes pas été le classique qu'Henry Jouin a cherché à tout prix à montrer. Son tempérament est profondément romantique et passionné et il ne recule devant aucun réalisme pour faire passer ses idées. Ses idées, et notamment ses conceptions politiques, ne pouvaient justement pas le laisser indifférent à la cause grecque. Fils de soldat républicain, lui-même républicain convaincu, impliqué en 1815 dans le carbonarisme italien, membre très tôt de la franc-maçonnerie, futur député de la République de 1848, David ne pouvait pas ne pas s'enthousiasmer pour un peuple se soulevant et luttant pour son indépendance. Son implication éventuelle dans les mouvements et associations philhellènes au cours de la guerre d'indépendance reste toutefois mal connue. Il est vrai que, comme l'ont rappelé certaines études ${ }^{36}$, la mobilisation des artistes est restée le plus souvent au stade de l'initiative artistique, se limitant à illustrer ou à célébrer tel ou tel épisode, en obtenant un impact plus ou moins marqué sur l'opinion.

On trouve toutefois une première trace de son engagement actif en février 1826. Elle est contenue dans une lettre à son ami d'Angers, le directeur de journal Louis Pavie ${ }^{37}$. David lui envoie un "prospectus pour les Grecs », en lui donnant le nom d'un habitant d'Angers " qui doit se charger de faire souscrire ». Et il ajoute : "à notre dernier dîner, nous avons tous souscrit. Il est question de cela dans La Pandore, je vous l'envoie. Vous verrez s'il est convenable que vous mettiez cet article dans votre journal ». La Pandore 
était alors l'un des principaux journaux parisiens consacré aux spectacles et aux arts. Le 13 février, un article y évoquait effectivement la mobilisation des citoyens de l'Anjou résidant à Paris ${ }^{38}$.

Autres signes d'un intérêt pour la cause grecque, les bustes des héros de la guerre d'indépendance qu'il veut réaliser en se rendant sur place - nous allons y revenir - ou les médaillons qu'il consacre aussi à ceux qui soutiennent la cause. Ces médaillons sont une vaste entreprise personnelle, poursuivie tout au long de sa carrière, pour fixer dans le bronze en plusieurs exemplaires, et ainsi les immortaliser, les traits des grands hommes de son temps ou de toutes celles et ceux qui lui semblent dignes de mémoire ${ }^{39}$. Il place dans son Panthéon quelques philhellènes de premier plan. Ainsi François Pouqueville (1770-1838), médecin, diplomate et écrivain, consul de France à Janina puis à Patras, auteur d'une célèbre et influente Histoire de la régénération de la Grèce, parue en 1824. Puis le fameux général Fabvier (1782-1855), modelé en 1828 avec son turban sur la tête au retour de Grèce, après l'épisode du siège de l'Acropole ${ }^{40}$. Le médaillon de JeanGabriel Eynard (1775-1863), surnommé «l'ami des Grecs », mais aussi « leur banquier », date, lui, de 1830. Quant à Byron, son médaillon est situé par Jouin vers 1838 et David ne l'a jamais rencontré ${ }^{41}$.

Le sculpteur ne semble pas avoir adhéré au principal comité philhellène qui se constitue à Paris en $1825^{42}$. Mais il s'inscrit le 2 janvier 1829 à la Société hellénique, créée en décembre, dont le but particulier est d'aider au développement de l'éducation en Grèce ${ }^{43}$. Certes toutes les associations philhellènes d'Europe ont eu cet objectif dans leurs statuts à des degrés divers. Mais après Navarin et l'expédition militaire de Morée, il était normal qu'une association nouvelle se concentre sur ce sujet, les buts militaires étant devenus désormais inutiles. La Société hellénique n'eut qu'une existence éphémère de deux années; elle fut victime de la décrue très rapide des mouvements philhellènes, engagée dès $1828^{44}$. Retenons néanmoins cet intérêt particulier de David pour la formation des Grecs : peut-être s'agit-il d'un signe avant-coureur du rôle qu'il s'imaginera par la suite pouvoir jouer personnellement sur place.

\section{Le voyage rêvé}

Quels contacts, justement, entretient-il avec des Grecs? Nous avons vu, en 1833, l'intervention de Michel Schinas. Ce dernier eut un rôle politique à son retour en Grèce, mais nous ignorons si David conserva des liens avec lui. Autre contact politique, plus important et sans doute plus durable, celui de Ioannis Kolettis, l'un des principaux protagonistes de la guerre d'indépendance, premier ministre du roi Othon avant d'être écarté, et nommé ambassadeur à Paris de 1835 à 1843, où il se lia d'amitié avec Guizot, ministre puis chef du gouvernement du roi Louis-Philippe. Kolettis sera de nouveau premier ministre grec de 1844 jusqu'à sa mort en 1847. En retour de la décoration qu'il lui a adressée en 1836, David réalise son médaillon, daté de la même année ${ }^{45}$. C'est lui qui pousse le sculpteur à venir s'installer en Grèce.

David eut assez tôt, semble-t-il, l'envie de visiter un pays occupant une place majeure dans son imaginaire ${ }^{46}$. Mais longtemps cette envie ne prit pas de vraie consistance. Les choses changent en 1841. C'est son ami Adrien Maillard qui en témoigne le premier en octobre devant une société savante de l'Anjou : « quand nous vîmes le statuaire David il y a peu de jours, il se disposait à quitter momentanément la France et à s'embarquer pour la Grèce ${ }^{47}$ ». Le projet réapparaît trois mois plus tard, dans une lettre de David à 
Victor Pavie, le 2 janvier 1842 : «M. Lenormant est de retour de Grèce depuis quelques jours seulement. Ce qu'il m'a raconté de son voyage me fait éprouver un profond regret de n'avoir pas pu exécuter le projet que j'avais formé ${ }^{48}$ ». Il s'agit de Charles Lenormant (1802-1859), archéologue, ancien membre de l'expédition de Morée, que Guizot avait nommé en 1830 chef de la section des Beaux-Arts au ministère de l'Intérieur et qui, à ce titre, avait attribué une première fois à David la réalisation du fronton du Panthéon. Devenu professeur d'histoire ancienne à la Sorbonne et membre de l'Institut, il venait d'être chargé par Guizot en 1841 d'une mission diplomatique, avec Jean-Jacques Ampère et Prosper Mérimée, pour étudier sur place l'influence de l'Angleterre, de l'Allemagne et de la Russie sur le gouvernement du roi Othon ${ }^{49}$. David continue :

«Il m'avait annoncé à plusieurs Grecs de mes amis qui occupent les premiers emplois dans ce pays. Enfin, rien n'est encore abandonné à l'égard de ce projet et l'hiver prochain, je puis aller m'établir à Athènes, où il reste encore de vieux défenseurs de la cause libérale qui attendent la consécration de mon ciseau ".

Réapparaît ici la vieille idée de David de faire les portraits des héros de l'indépendance encore en vie. Nous savons par exemple qu'en 1836, il cherche à se procurer auprès du fils de Constantin Canaris résidant à Paris, un portrait de son père ${ }^{50}$. La suite de la lettre donne un autre but au projet de voyage :

« au mois de septembre, je porterai mes regards et mon cœur vers cette chère Grèce que j'ai si longtemps désiré voir, l'ambassadeur grec et tous mes amis qui habitent ce pays m'engagent depuis longtemps à aller m'y fixer pour y ouvrir une école de sculpture ».

On voit donc se dessiner ici une sorte de réseau qui pousse David vers la Grèce : Lenormant, qui au retour de sa mission, veut renforcer la présence française en Grèce et Kolettis qui œuvre dans le même sens, le tout sans doute sous l'œil bienveillant de Guizot.

Il y a certes une part de dépit dans le projet de David; il estime son art de moins en moins reconnu en France « qui ne comprend plus le sentiment de patriotisme » comme il le dit à Pavie ${ }^{51}$. En Grèce, cela ne peut être que différent... Et son projet est sérieux. Le 19 juin, il écrit à son ami qu'il n'a pas encore pu quitter Paris en raison d'une grave maladie de son fils Robert. Mais la santé du garçon s'améliorant, il pense pouvoir partir bientôt. Il a d'ailleurs pris ses dispositions. Il a fermé son atelier et confié ses élèves à François Rude ; il a rédigé son testament, comme il le fait à chaque fois qu'il part pour un long voyage : «sous peu de jours, je crois pouvoir partir. Je vais encore éprouver de grandes et saisissantes impressions à la vue des beautés de ce pays méridional que j'aime tant ». Mais c'est par une interrogation où perce comme une pointe d'angoisse qu'il termine sa lettre : « Mais toi [...] viendras-tu me retrouver en Grèce $?^{52}$ ».

Finalement, le voyage ne se fera pas, sans raison connue. Le 27 juin, il écrit que Robert est toujours malade ${ }^{53}$. Puis il part en août pour une cure thermale dans les Pyrénées. Est-ce sa santé fragile? Ou s'agit-il de commandes en retard qu'il doit livrer? Nous l'ignorons.

\section{III - 1852 : Le séjour en Grèce ou le rêve brisé}

C'est une décennie plus tard que le rêve devint réalité pour David, mais dans des circonstances qu'il n'avait certainement pas imaginées, celles de la proscription et de l'exil. Ses dispositions d'esprit ne pouvaient en être ni sereines, ni positives. 
êté au moment du coup d'état de décembre 1851, l'ex-député républicain est rapidement relâché, mais condamné à l'exil. Il s'installe à Bruxelles avec sa famille, mais pour tromper son chagrin et son ennui, il décide peu après de voyager. Il repense alors à la Grèce. Pour Henry Jouin, il a toujours en tête son projet «d'une école d'art dans la patrie de Phidias. Il comptait y former des élèves. Quelques jeunes Grecs avaient autrefois suivi son cours, David essayerait de les rejoindre et de les grouper autour de $l^{1} i^{54}$ ». Malheureusement, Jouin n'en dit pas davantage. Qui sont ces « jeunes Grecs » qui "avaient autrefois suivi son cours»? Vraisemblablement des étudiants inscrits à l'École des Beaux-Arts où il enseigna à partir de 1826; mais pas de noms grecs dans les listes connues des élèves de son atelier, l'un des plus importants de la première moitié du siècle.

Il arrive à Athènes au début du mois de mai 1852, accompagné de sa jeune fille Hélène. À l'excitation des premiers jours, provoquée par la nouveauté de la lumière, la beauté des paysages, le pittoresque de certaines scènes et l'idée de parcourir des sites célèbres, succède très vite la déception la plus amère et la plus noire. La chaleur croissante, très éprouvante pour cet homme déjà âgé (il a 64 ans) et en mauvaise santé, la rudesse des mœurs des habitants, la pauvreté ou même la misère partout présente qui le frappent beaucoup, le désintérêt qu'il croit constater pour les témoignages du passé, l'accablent, le découragent et entretiennent son mal du pays. Il suffit de citer un passage de ses écrits, car ils sont tous sur le même ton et souvent plus sévères :

"Quelle dérision! Chaque rue d'Athènes porte le nom d'un grand homme de l'Antiquité! Sur des piédestaux, dont les statues ont été détruites ou enlevées, on voit des Grecs modernes gravement assis, cherchant à se débarrasser de la vermine qui les ronge. On vend de la friture et des haillons sur les autels des dieux, les noms héroïques de Thémistocle et de Miltiade se lisent sur les devantures d'un parfumeur ou d'un épicier et pour couronnement à cet ensemble ironique, le Parthénon en ruine conserve quelques colonnes debout; on dirait les bras d'un squelette implorant le ciel ${ }^{55} \%$.

Il constate rapidement le décalage de son projet d'école, avec la réalité qu'on lui montre :

«aujourd'hui [ce passage des carnets n'est pas daté, mais se place en mai ou juin] j'ai été appelé par le directeur de l'École des Beaux-Arts à venir juger le concours des élèves. C'est d'une nullité effrayante. Ce sont tous des enfants de la classe la plus malheureuse de la société. On leur donne leur prix en argent et cela sert à faire vivoter la famille. Aussi, ils ne peuvent faire aucune étude sérieuse. Et puis quels professeurs, bon Dieu! C'est la nullité des nullités. Mais maitres et élèves sont enchantés et pleins de confiance en eux $!^{56} \%$.

41 On observe ici que le républicain David n'a plus aucun recul, ne cherche plus à comprendre, ni à excuser. Il ne reste que la déception la plus brutale et sans appel.

Il renonce donc très vite à son projet d'école, malgré le bon accueil qu'il reçoit à la fois de la part des Grecs et de la communauté française. Les élèves de la toute jeune école française, Edmond About (1828-1885) et Charles Garnier (1825-1898) de retour d'Égine ${ }^{57}$, mais aussi le peintre Alfred de Curzon (1820-1895), viennent à sa rencontre et s'émeuvent de son sort de proscrit. Quand est connue la mort du sculpteur Pradier, intervenue en France le 5 juin, Edmond About saisit l'occasion et envoie un article au journal L'Illustration qui paraît le 27 juillet à Paris. Il veut attirer l'attention sur le sort de David : «Pradier est mort, écrit-il, et le jour où la France se voit enlever l'un de ses deux grands sculpteurs, il est assez naturel qu'elle se demande où est l'autre ». Et de comparer ensuite l'exil de David à celui de Phidias à Olympie ${ }^{58}$. Le ton de l'article et son 
contenu ne sont pas du tout du goût des autorités françaises et manquent de causer le renvoi d'About. Il fallut, pour l'éviter, l'intervention de Joseph-Daniel Guigniaut (1794-1876), influent helléniste français bientôt professeur au Collège de France et l'un des pères fondateurs de l'École française d'Athènes ${ }^{59}$.

David rencontre aussi Charles-Ernest Beulé (1826-1874) qui fouille sur l'Acropole. Beulé, dans son Journal de fouilles, a évoqué a posteriori cette rencontre avec talent, en cernant bien la disposition morale et physique du vieil artiste :

«Il est un visiteur cependant qui m'a ému à la première vue, avant même que j'eusse appris qu'il était un artiste illustre et un proscrit. Je l'avais remarqué à diverses reprises mais avec son visage flétri par le chagrin, sa moustache épaisse, son paletot clair et boutonné, il ressemblait à un Polonais. Il s'appuyait sur le bras d'une jeune fille, si pâle elle-même qu'elle aurait eu plutôt besoin d'appui ; tous deux gravissaient péniblement le rocher de l'Acropole, s'asseyaient sur les marches du Parthénon, contemplaient tristement la mer et redescendaient non sans s'être un peu ranimés devant la frise de Phidias. Je songeais involontairement à đdipe et Antigone [...]. Un jour, le voyageur [...] m'adressa sur mes fouilles quelques questions auxquelles je répondis avec le désir de le satisfaire. Il me remercia, me tendit la main et me dit qu'il s'appelait David d'Angers ${ }^{60}{ }^{\prime}$.

Parmi les Grecs qu'il rencontre et qui lui font bon accueil, Henry Jouin cite ${ }^{61}$ Kyriakos Pittakis (1798-1863), premier éphore des antiquités d'Athènes et combattant de la guerre d'indépendance ; Alexandros Rangavis (1809-1892), connu en France sous le nom de Rangabé, homme de lettres, philologue, archéologue, mais aussi homme politique qui fut ministre et ambassadeur. Il cite les Soutzo, les Caradja, sans plus de précisions... Il mentionne encore la duchesse de Plaisance, Sophie de Marbois (1785-1854), installée en Grèce après 1830. Elle lui aurait commandé, selon Beulé, un bas-relief pour l'une de ses demeures, à placer au-dessus de la porte d'entrée, sur le sujet de Thémistocle chez Admète, image de l'hospitalité ${ }^{62}$.

David tente de trouver un dérivatif dans le travail. Il peut enfin faire le buste de Constantin Canaris (1790-1877), qui est le type même du héros qu'il vénère. La galerie d'Angers en conserve le modèle (fig. 6). Écoutons ce qu'en dit Henry Jouin :

«Le buste de Canaris est de proportions colossales. Le brûleur de flottes porte la veste grecque, l'écharpe nouée avec négligence autour du cou, le bonnet de pêcheur sur la tête. Au premier aspect, le laisser-aller du portrait trahit le caractère personnel du marin d'Ipsara. [...] Aventurier de génie, Canaris porte écrit sur ses traits la rudesse de son courage, le front haut et large, les joues sèches, le regard d'une fixité terrible [...], les rides nerveuses de la face indiquent la puissance de volonté ${ }^{63}$ \%.

Ce sera le dernier buste modelé par David: il y fait preuve d'une liberté de facture étonnante ; il soumet tout à l'idée qu'il veut donner de son héros, tout en le rendant parfaitement reconnaissable. 
Figure 6.

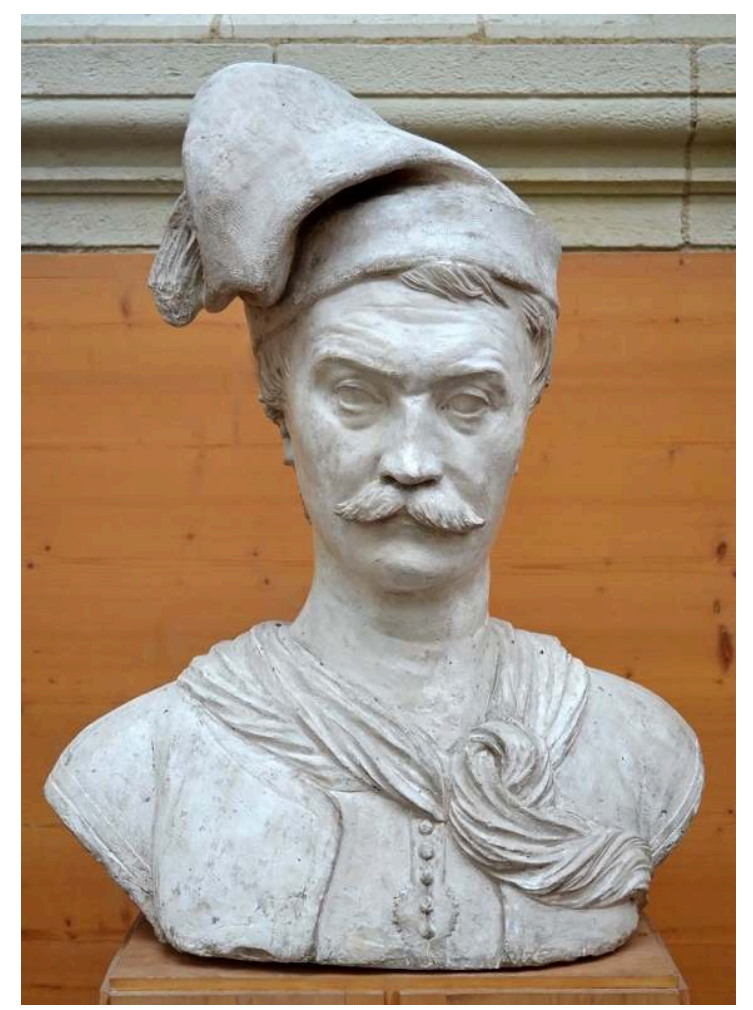

David d'Angers, Buste de Constantin Canaris, Angers, Galerie David d'Angers, inv. MBA 856.25.

(c) Wikimedia Commons

47 La réalisation du buste ne passe pas inaperçue à Athènes. Le journal Nea Pandora en publie une gravure ${ }^{64}$ et Edmond About - qui exagère sans doute - indique dans son article pour L'Illustration que «toute la ville s'occupe de David d'Angers et du buste de Canaris...». Il donne d'intéressantes précisions sur les conditions dans lesquelles travaille le sculpteur, qui éclairent sans doute assez bien les raisons de son renoncement pour le projet d'école :

«M. David [...] n'aurait pas su où travailler sans l'obligeance du docteur Reuser, médecin du roi [...]. Par une de ces plaisanteries que le sort se permet quelques fois, c'est dans l'hôpital des aveugles que M. David a fait son buste. L'atelier trouvé, il fallait se procurer de la terre [...]. La glaise ne valait rien; elle était courte et se crevassait tous les jours, quelque soin qu'on prit de la mouiller. Le modèle achevé, pas de mouleurs : ce sont les élèves de l'école de sculpture qui ont fait le moule ; mais le plâtre n'était pas meilleur que la terre; il a fallu travailler sur nouveaux frais. Enfin, le buste est fait, et le marbre viendra bientôt, mais il faudra que M. David se serve lui-même de praticien ${ }^{65}$ ".

Il n'est pas sûr, en fait, que David ait eu le temps de réaliser ce marbre ${ }^{66}$. En revanche, il modèle aussi le médaillon de Canaris et celui de sa femme Despina. Dans ses carnets, il dit : «Je savais que cela le toucherait profondément, qu'elle était la digne femme d'un héros $^{67}$ ». À cette belle femme, il donne un profil grec classique. Mais il refuse d'être présenté au roi ${ }^{68}$.

Dès juillet, il a pris la décision de repartir. En attendant, il tente d'échapper un peu aux chaleurs de l'été en résidant à Cephisia, sur les flancs du Pentélique. Il en visite les carrières, va sur le site de Marathon et à Thèbes. Il fait un bref voyage d'une semaine, en octobre semble-t-il, à Smyrne et à Constantinople ${ }^{69}$. Il rentre à Athènes, mais avant 
de partir s'installer à Nice, qui à l'époque n'est pas encore territoire français, il décide de passer par Missolonghi pour revoir sa Jeune Grecque. On tente de l'en dissuader car les mutilations qu'elle a subies sont connues à Athènes. Beulé s'en est fait l'écho dans son journal :

«Aujourd'hui, David m'a abordé avec une sorte de fureur. On lui a dit que le monument qu'il avait exécuté jadis pour le tombeau de Botzaris était mutilé, que les habitants de Missolonghi s'en servaient comme de cible, que la statue de sa Jeune Grecque était criblée de grains de plombs. - J'irai à Missolonghi, je m'assurerai de mes propres yeux et, si le fait est vrai, j'écrirai aux journaux pour dénoncer la Grèce au mépris de l'Europe. - J'essaye en vain de le calmer, mais les Grecs sauront le désarmer; ils sont trop fins pour ne pas racheter leur négligence. Si David va en effet à Missolonghi, les habitants qu'on avertira d'Athènes l'accueilleront avec de telles manifestations d'enthousiasme, qu'il sera forcé de leur pardonner ${ }^{70}$ ».

En fait, David ne pardonnera pas, même si la suite de l'histoire paraît s'être déroulée à peu près comme le prévoyait Beulé. Quittant Athènes vers le 15 novembre, David passe par l'isthme de Corinthe, prend le bateau, s'y voit abordé par un juge d'origine italienne se rendant aussi à Missolonghi, qui tente de le dissuader, avant de lui avouer qu'il est missionné pour le faire ${ }^{71}$... À l'arrivée, David se précipite voir sa statue :

«Je viens de voir le monument de Marco Botzaris, il est presque auprès du bastion où il a été tué [les informations dont dispose David sont fausses : Marco Botzaris est mort à Karpenisi, des suites de la blessure à la tête reçue lors de l'attaque du camp turc]. Le piédestal est de forme égyptienne et très élevé. Tout auprès est un tumulus qui couvre les ossements des braves tués auprès de lui. De très loin, j'ai aperçu la statue de la jeune fille qui regarde son nom sur la pierre tombale, il m'a semblé voir ce jeune enfant tressaillir en voyant son créateur d'il y a trente années. Les Grecs lui ont cassé les mains, le doigt qui épelait et un pied, ainsi que les deux oreilles... ${ }^{72}$ ».

51 Suivent des considérations désespérées sur la nature humaine et l'ingratitude barbare des Grecs. Son sentiment est renforcé par le fait qu'il ne parvient pas à retrouver la tombe de Byron, ni la maison où il est mort, dont on lui dit qu'elle a disparu et qu'elle a été remplacée par une autre... Une délégation municipale conduite par le maire vient lui lire une pompeuse déclaration de remerciement et de louange, traduite par le médecin bavarois qui l'héberge. Il y est comparé à Phidias et à Praxitèle ce qui, osons l'expression, le laisse de marbre. Il repart, dégoûté, au bout de trois jours. "J'ai dit ce matin adieu à ma pauvre petite mutilée du monument de Botzaris ; elle est à l'image de la réputation des hommes qui, même après bien des années sont la victime de la fureur jalouse, de l'envie qui s'attache à leur mémoire ${ }^{73}$ ». On lui avait raconté en effet que la statue avait été victime d'une bande de pallikares ${ }^{74}$ d'un clan rival de celui de Botzaris. En fait, plusieurs versions différentes ont circulé quant à l'origine des dégradations, sans qu'il soit aujourd'hui encore possible d'y voir vraiment clair.

\section{IV - Le destin d'un monument}

Arrivé à Nice en décembre, épuisé et malade, David fut autorisé trois mois plus tard à rentrer en France. C'était la fin de son exil et de son aventure grecque; il en gardait une blessure ayant nom La Jeune Grecque. Que devint la statue? On peut pour cela laisser la parole à Henry Jouin : "Les mutilations de La Jeune Grecque furent connues en France par quelques phrases de $\mathrm{M}$. About dans La Grèce contemporaine, dont la première édition date de 1854. Le livre et l'homme furent sévèrement jugés à Athènes après cette dénonciation. La presse donna quelque retentissement à l'affaire, dont le souvenir se 
trouva de nouveau ravivé à la mort de David [le 6 janvier 1856]. C'est alors que le gouvernement grec conçut la pensée singulière de faire transporter au musée d'Athènes le marbre mutilé et voulut obtenir un bronze de la Jeune Grecque, soit à l'aide d'une copie, soit d'après le modèle en plâtre déposé au musée David ». Contactée le 3 septembre 1858 par le chargé d'affaires grecques à Paris,

«Mme David s'opposa formellement à ce projet, voulant éviter que le nom de son mari figurât sur une œuvre qui ne serait pas sortie de ses mains ; mais elle engagea le chargé d'affaires à demander l'envoi en France du marbre orignal, qu'un élève de David, Armand Toussaint, essayerait de réparer en s'aidant du modèle en plâtre, d'ailleurs trop imparfait pour être fondu. Les pourparlers furent très longs. Enfin, la Jeune Grecque arriva subitement à Paris dans une caisse où elle n'était protégée d'aucune façon contre les chocs d'une aussi longue traversée ${ }^{75} »$.

Toussaint étant mort le 24 mai 1862, c'est finalement un autre élève de David, JeanJules Allasseur (1818-1903) qui réalisa et termina en 1866 la restauration que nous voyons aujourd'hui. Allasseur n'a sans doute pas pu trouver le même marbre légèrement veiné et tacheté venant des Pyrénées que David avait utilisé ${ }^{76}$, en voulant affirmer un caractère français à sa statue et en n'utilisant pas l'habituel marbre de Carrare. Cela pourrait expliquer le blanc plus prononcé du marbre utilisé par Allasseur, sans doute de Carrare... Remarquons ici que l'œuvre gagnerait à ce qu'on puisse tourner autour d'elle, comme le permet la présentation du plâtre à Angers (fig. 7).

Figure 7.

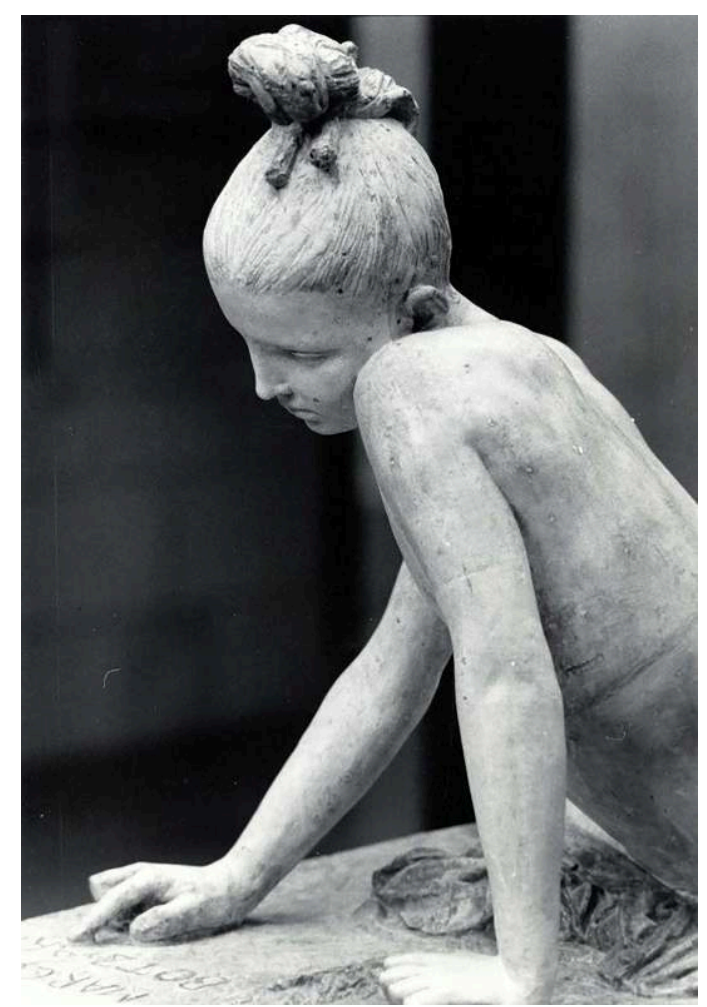

David d'Angers, La Jeune Grecque, détail, Angers, Galerie David d'Angers, inv. MBA 856.15 (c) Philippe Durey

54 L'œuvre repartit tout de suite pour Athènes, ne retourna pas à Missolonghi, mais resta semble-t-il entreposée au musée archéologique. À partir de 1884, elle fut à nouveau visible $^{77}$, exposée en divers lieux par les soins de la Société historique et ethnologique 
de Grèce, chargée de créer le musée national d'histoire. Voilà La Jeune Grecque exposée en 1896 dans l'École polytechnique (fig. 8). À partir de 1962, elle trouva sa destination finale dans l'ancien Parlement, devenu le cadre de l'actuel musée historique. Elle y reçut en 1963 la visite du général de Gaulle ${ }^{78}$. Cela n'empêcha pas que trois ans plus tard, lors d'une exposition consacrée à David d'Angers à l'Hôtel de la Monnaie à Paris en 1966, l'œuvre, perdue de vue par les spécialistes français, ne soit considérée dans le catalogue comme purement et simplement détruite ${ }^{79}$. Ce qui valut au conservateur du musée d'Angers, André Bruel, de recevoir une lettre de mise au point ferme de son homologue du musée historique national, Jean Mélétopoulos ${ }^{80}$.

Figure 8.

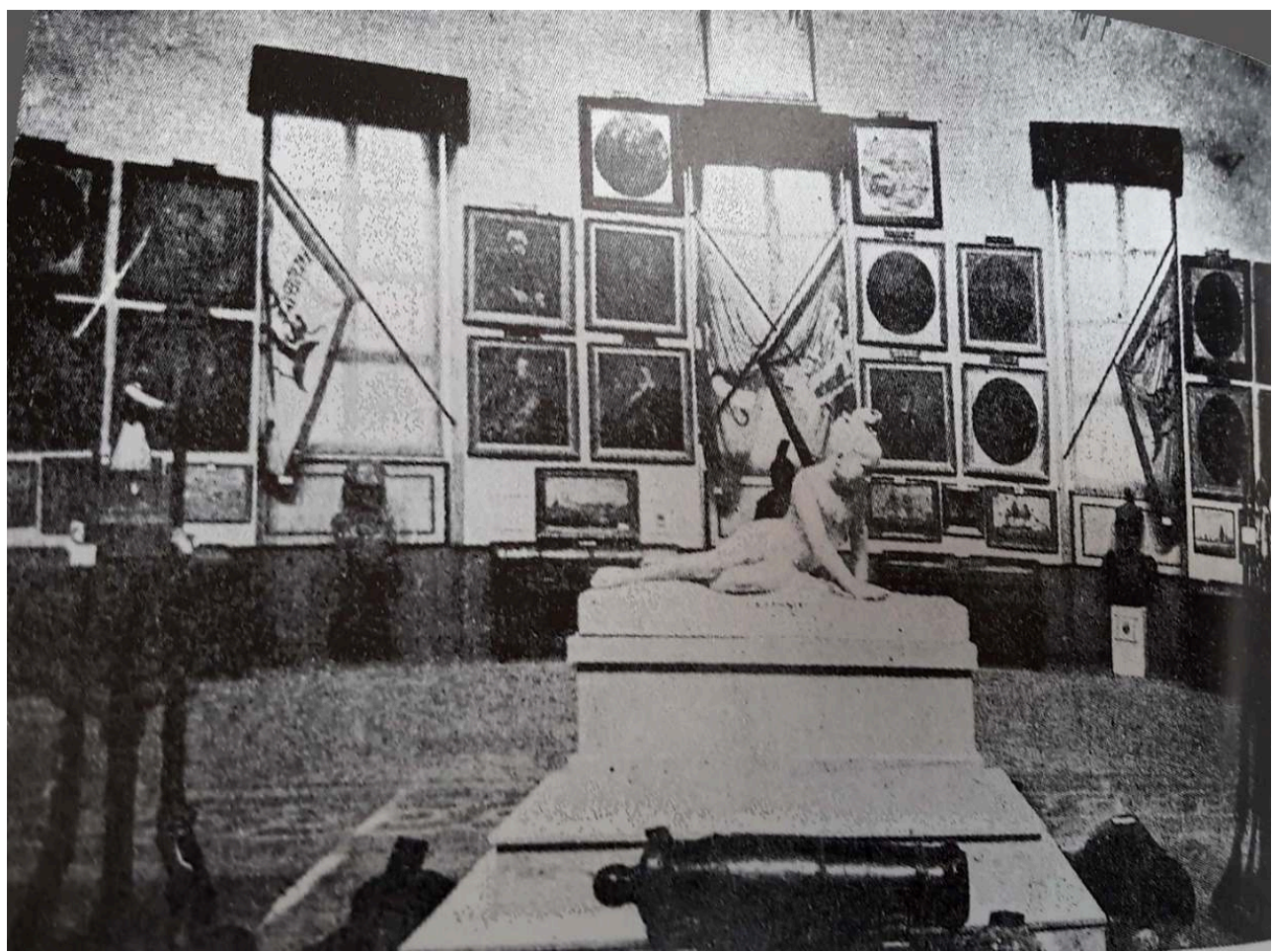

La Jeune Grecque exposée en 1896 dans l'École polytechnique, Geroyannis-Petimezas, « La Présence de David d'Angers en Grèce », p. 186.

Il y a, malheureusement, davantage qu'une anecdote dans cette erreur grossière à cette date concernant une œuvre tout de même majeure de la sculpture française du $\mathrm{XIX}^{\mathrm{e}}$ siècle. Cet art et ses productions sont alors en France au creux de la vague et même assez largement méprisés. Si David intéresse à l'époque, c'est par ses écrits et sa correspondance avec l'Europe intellectuelle de son temps, et par les portraits qu'il en réalise. Le réveil des galeries de sculpture du XIX siècle n'interviendra en France qu'une vingtaine d'années après l'exposition de l'Hôtel de la Monnaie. La réinstallation de celle de David à Angers en 1984 en sera l'un des premiers signes, peu de temps avant l'ouverture du musée d'Orsay.

On peut penser finalement que David serait aujourd'hui secrètement heureux que l'un de ses chefs-d'œuvre, "sa chère enfant", soit à Athènes à proximité des trésors antiques de l'Acropole magnifiquement remis en valeur, et parmi les souvenirs de la guerre d'indépendance. En 1852, compte tenu de l'état d'esprit qui était le sien, compte tenu de sa recherche illusoire d'une terre idéale faite uniquement d'héroïsme et de 
beauté, capable de lui faire oublier ses déceptions morales et politiques de proscrit, son voyage ne pouvait pas réussir ; le rêve du vieux sculpteur ne pouvait qu'être brisé.

\section{NOTES}

1. Ce texte est celui d'une conférence donnée à l'École française d'Athènes le 6 juin 2018. Je remercie vivement Alexandre Farnoux, alors son directeur, de m'avoir invité à la prononcer et d'avoir autorisé, en accord avec Claire Barbillon, sa publication dans le cadre de cette revue, suite à la reprise de cette conférence à l'École du Louvre le 8 novembre 2018.

2. Georges Geroyannis-Petimezas, "La Présence de David d'Angers en Grèce ", Marie-Rose et Marguerite-Cécile Albrecht, David d'Angers, regards autour d'un sculpteur, Maulévrier, Hérault éditions, 1987, p. 186 et p. 193, note 11. Bonanos fut élève de l'École des Beaux-Arts d'Athènes et se forma aussi à Rome. Il est l'auteur de nombreux marbres illustrant aussi bien des sujets mythologiques que des personnages de l'histoire grecque moderne.

3. Sur les significations multiples que David donnait à sa statue, voir son propre texte : « Une nuit d'atelier ", Henry Jouin, David d'Angers, sa vie, son cuvre, ses écrits et ses contemporains, 2 vol., Paris, E. Plon et $\mathrm{C}^{\mathrm{ie}}, 1878$, tome II, Mélanges, pp. 345-346. Voir aussi les interprétations de Jouin dans le tome I, pp. 173-176.

4. H. Jouin, op. cit. note 3 [1878], II, pp. 344-345.

5. Anonyme [Adèle Hugo], Victor Hugo raconté par un témoin de sa vie, 2 vol., Paris, Lacroix Verboeckhoven, 1863, tome 2, pp. 225-226. Cité par Jouin, op. cit. note 3 [1878], I, pp. 173-174.

6. H. Jouin, David d'Angers et ses relations littéraires. Correspondance du maitre, Paris, E. Plon, Nourrit et $C^{\text {ie }}, 1890$, pp. 26-27. Lettre de David à Pavie père, 22 décembre $1827:$ « Je suis toujours enchanté que vous m'ayez fait connaître Hugo ».

7. Registre du Salon de 1827, Enregistrement des ouvrages, Archives nationales, fonds Archives des musées nationaux, côte 20150431/26 ( ${ }^{*}$ KK 25).

8. La bataille de Navarin eut lieu le 27 octobre et l'inauguration du Salon le 4 novembre. Eva Bouillo (Le Salon de 1827, classique ou romantique ? Presses universitaires de Rennes, 2009, pp. 59-60 et annexes 2 et 3, p. 257) a recensé 23 « sujets grecs » dont 15 peintures d'histoire.

9. Journal des Artistes, 9 mars 1828, p. 151 : «M. David, dont nous regrettions de ne pas voir le Racine, vient de le faire placer dans la salle d'exposition ». C'est sur le Racine que se concentrent les commentaires des œuvres exposées par David; voir E. Bouillo, op. cit. note 8, annexe 16, pp. 269-270.

10. H. Jouin, op. cit. note 3 [1878], I, p. 180 et suivantes.

11. David signe ses œuvres en utilisant son seul patronyme, sans y ajouter «d'Angers ». Cette mention, indiquée de surcroit ici avec de surprenantes parenthèses, ressemble fort, malgré l'usure que nous avons pu observer sur place, à un ajout postérieur assez maladroit.

12. Un bel exemplaire à patine brune passé en vente à la galerie André Lemaire à Paris (avant 2014), signé et daté sur la tranche de la terrasse : «P. J. David/1834», inscrit sur la terrasse «F. Barbedienne fondeur», cachet « réduction mécanique Collas» sur la tranche de la terrasse, H. 21,5 cm. Florence Rionnet (Les Bronzes Barbedienne, l'œuvre d'une dynastie de fondeurs (1834-1954), Paris, Arthena, 2016), reproduit l'exemplaire conservé au musée Fabre à Montpellier (p. 180, fig. 194), sans mentionner de date, et indique dans son catalogue (p. 296, cat. 571) des éditions proposées dès 1847, réalisées à partir de 1855 et poursuivies jusqu'en 1911. 
13. G. Geroyamis-Petimezas, op. cit. note 2, image IV.

14. Jean Brihault, «La France choisie de Lady Morgan », Paul Brennan et Michael O’Dea (dir.), Entrelacs franco-irlandais: Langue, mémoire, imaginaire, Caen, Presses universitaires de Caen, 2004, $\S 37$ [en ligne : http://books.openedition.org/puc/1191].

15. Lady Morgan, La France en 1829 et 1830, traduit de l'anglais par A. Sobry, Paris, H. Fournier Jeune libraire, 1830, tome 1, pp. 262-263.

16. Cité par H. Jouin, op. cit. note 3 [1878], I, p. 173, note 1.

17. H. Jouin, op. cit. note 3 [1878], II, p. 345.

18. André Bruel, Les Carnets de David d'Angers, 2 vol., Paris, Plon, 1958, tome 2, pp. 409-410. Dans une lettre du 10 janvier 1853 à Victor Pavie, il reprend et développe ce thème : «Du plus loin que je découvris ma pauvre petite, mon cœur palpita violemment. Une espèce d'hallucination me laissa croire que, triste et plaintive, elle me faisait reproche de l'avoir exilée au milieu de Barbares. Nous avions vécu si longtemps ensemble ! Je m'imaginais que le marbre s'était pénétré des émotions qui m'avaient agité tant de nuits quand je terminais cette forme de ma pensée. » : Henry Jouin, op. cit. note 6 [1890], p. 309. Ce genre de reprise et de variante sur un même thème est très fréquent pour les écrits de David, entre sa correspondance, ses «notes " telles que Jouin les a retranscrites et parfois modifiées, et les 54 carnets dont elles proviennent en grande partie et qu'André Bruel a publiés intégralement; sur ce sujet, voir Jacques de Caso, David d'Angers, l'avenir de la mémoire, Paris, Flammarion, 1988, pp. 48-49.

19. Il a été donné au musée d'Angers par $\mathrm{M}^{\mathrm{me}}$ David en 1856.

20. Lettre du 14 août 1834, publiée par H. Jouin, op. cit. note 6 [1890], p. 81.

21. Ferdinand Letessier, "Chateaubriand et David d'Angers ", Bulletin de l'Association Guillaume Budé, 1953, LH-12, pp.131-132, publie la courte réponse de Chateaubriand, conservée à la Bibliothèque municipale d'Angers, à l'invitation reçue de David. Notons ici que le célèbre poème, L'Enfant grec, que Marceline Desbordes-Valmore a consacré à cette sculpture, vue par elle vraisemblablement dans l'atelier, («Ce gracieux enfant, cette innocence nue / qui se prend à rêver au marbre d'un tombeau... »), apparait, dans une première version, intitulée La Jeune fille au tombeau de Botzaris, dans une lettre qu'elle adresse à David le 13 mai 1833 (publiée par Arthur Pougin, La Jeunesse de Madame Desbordes-Valmore, Paris, C. Levy, 1898, p. 196). Le poème fera ensuite partie, en 1839, du recueil Pauvres Fleurs (Paris, Dumont Éd., p. 105).

22. Anonyme [F.] Journal des Artistes, 1834, vol. II, 17 août, pp. 104-106.

23. Pittoresque auquel n'échappe pas, par exemple, John Étienne Chaponnière (1801-1835) avec sa Jeune Grecque (ou jeune captive) pleurant sur le tombeau de Byron, exposée à Genève en 1827, plâtre acquis la même année par le Comité grec de Genève pour le musée Rath, aujourd'hui au musée d'Art et d'Histoire ; voir Claude Lapaire, "La Sculpture à Genève au XIX siècle - questions de méthode ", Revue suisse d'art et d'archéologie, 38, 1981, p. 104.

24. Gustave Planche, Études sur l'école française (1831-1852), peinture et sculpture, Paris, Michel Lévy Frères,1855, vol. I, «Salon de 1834 », pp. 271-272 : «pourquoi tout cela n'est-il pas venu au Louvre? Ce n'est pas assez de laisser voir ses ouvrages à quelques amis d'élite, ou à quelques curieux privilégiés [...]».

25. H. Jouin, op. cit. note 3 [1878], I, p. 172.

26. Terme dérivant de Phanari, quartier de Constantinople, et désignant un Grec membre de la couche sociale participant, à partir du XVII ${ }^{\mathrm{e}}$ siècle, au système administratif de l'empire ottoman. 27. Sur Schinas, voir Sophie Basch, Le Mirage grec : la Grèce moderne devant l'opinion française depuis la création de l'École d'Athènes jusqu'à la guerre civile grecque (1846-1946), Paris, Hatier, 1995, pp. 65 et 66 , note 48.

28. G. Geroyannis-Petimezas, op. cit. note 2, p. 181.

29. Id., p. 182.

30. Id., p. 182.

31. Id., p. 184.

Les Cahiers de l'École du Louvre, 16 | 2021 
32. H. Jouin, op. cit. note 6 [1890], pp. 105-106.

33. Journal des Beaux-Arts et de la Littérature, 17 juillet 1836, p. 46 : « Nouvelles ».

34. G. Geroyannis-Petimezas, op. cit. note 2, p. 183.

35. Id., p. 183.

36. Denys Barau, « La Mobilisation des philhellènes en faveur de la Grèce, 1821-1829 ", Populations réfugiées, de l'exil au retour, Luc Cambrézy et Véronique Lassailly-Jacob (dir.), Marseille, IRD Éditions, 2001, pp. 37-75, notamment pp. 45-48.

37. Lettre du 16 février 1826 à Pavie père, publiée par H. Jouin, op. cit. note 6 [1890], pp. 17-19.

38. «Souscription angevine ", La Pandore, Journal des spectacles, des lettres, des arts, des mœurs et des modes, $\mathrm{n}^{\circ} 1002,13$ février 1826, pp. 2-3.

39. Sur le sujet, voir cat. d'exp., David d'Angers, les visages du romantisme, sous la direction de Thierry Laugée et Inès Villela-Petit, Paris, BnF, Cabinet des médailles, Gourcuff Gradenigo, 2011.

40. Fabvier dirigea, de décembre1826 à juin 1827, une héroïque défense de l'Acropole assiégée par les Turcs.

41. Byron mourut à Missolonghi d'une affection pulmonaire le 19 avril 1824 .

42. La « Société philanthropique en faveur des Grecs ", dirigée par un comité de 25 personnalités, le « comité grec de Paris ».

43. H. Jouin, op. cit. note 6 [1890], p. 105. Denys Barau (op. cit. note 36, pp. 58-59) évoque ainsi cette Société hellénique: "Le même souci philanthropique d'édification - aux deux sens du mot d'une Grèce nouvelle préside en décembre 1828 à la création à Paris d'une Société hellénique pour la propagation des connaissances utiles en Grèce, à laquelle participent parmi de nombreuses personnalités du monde artistique, littéraire et universitaire, plusieurs membres du comité grec de Paris ».

44. D. Barau, op. cit. note 36, p. 44.

45. Et non de 1830, comme l'indique à tort H. Jouin, op. cit. note 6 [1890], p. 106.

46. Les premières traces de l'idée d'un voyage en Grèce figurent dans les lettres qu'il adresse en janvier 1815 à Louis Dupré : « Lettres de Pierre Jean David d'Angers à son ami Louis Dupré, peintre du roi de Westphalie » éditées par Robert David d'Angers, Paris, E. Charavay, 1891, p. 12 (lettre du 3 janvier) et pp. 18-19 (lettre du 20 janvier).

47. H. Jouin, op. cit. note 3 [1878], I, p. 417.

48. H. Jouin, op. cit. note 6 [1890], p. 190.

49. Sabine Jaubert, notice "Charles Lenormant ", Dictionnaire des historiens de l'art actifs en France de la Révolution à la Première guerre mondiale, Philippe Sénéchal et Claire Barbillon (dir.), Paris, INHA, 2020, [en ligne: https://www.inha.fr/fr/ressources/publications/publicationsnumeriques/dictionnaire-critique-des-historiens-de-l-art/lenormant-charles.html?search-

keywords=lenormant].

50. H. Jouin, op. cit. note 3 [1878], II, p. 381.

51. Lettre du 2 janvier 1842 : «Qui peut me retenir dans cette France, au milieu d'un peuple énervé qui ne comprend plus le sentiment de patriotisme?»; H. Jouin, op. cit. note 6 [1890], p. 190.

52. H. Jouin, op. cit. note 6 [1890], pp. 200-203.

53. H. Jouin, op. cit. note 6 [1890], p. 203.

54. H. Jouin, op. cit. note 3 [1878], I, p. 459.

55. Notes autographes citées par H. Jouin, op. cit. note 3 [1878], I, pp. 463-464. Ces images cruelles des rues d'Athènes reviennent dans une lettre à Victor Pavie le 3 juillet ; H. Jouin, op. cit. note 6 [1890], pp. 299-300.

56. A. Bruel, op. cit. note 18, II, p. 381.

57. Charles-Ernest Beulé, Fouilles et découvertes résumées et discutées en vue de l'histoire de l'art, vol. I, Grèce et Italie, Paris, Didier, 1873, "Journal de mes Fouilles », 25 mai 1852, p. 40 : "J'attends 
Garnier, il est dans l'île d'Égine avec About. L'un mesure les ruines du temple dorique [...] ; l'autre explore l'île et fait son noviciat scientifique ».

58. H. Jouin, op. cit. note 3 [1878], I, p. 469.

59. H. Jouin, op. cit. note 3 [1878], I, p. 470.

60. Ch.-E. Beulé, op. cit. note 56, 12 mars 1853, p. 67.

61. H. Jouin, op. cit. note 3 [1878], I, p. 468.

62. Ch.-E. Beulé, op. cit. note 56, 12 mars 1853, p. 68.

63. H. Jouin, op. cit. note 3 [1878], I, p. 467.

64. Année 1852, p. 231 : voir G. Geroyannis-Petimezas, op. cit. note 2, p. 188, reproduite image XII.

65. H. Jouin, op. cit. note 3 [1878], I, p. 470, note 1.

66. En 1987, Georges Geroyannis-Petimezas indiquait à ce sujet (op. cit. note 2, p. 188) avoir «commencé des recherches en collaboration avec les descendants de l'amiral»; nous ne connaissons pas, à ce jour, de mention de l'existence du marbre ni de son éventuel emplacement.

67. A. Bruel, op. cit. note 18, II, 24 juin, p. 377.

68. Id., p. 377 : «Depuis plus d'un mois que je suis à Athènes on a fait toutes les démarches pour m'engager à me faire présenter au Roi. Mes rois, ce sont les hommes de génie, dont les œuvres ont été utiles au progrès de l'humanité, et les braves qui ont combattu pour la liberté et l'indépendance de la patrie ».

69. H. Jouin, op. cit. note 3 [1878], I, p. 478.

70. Beulé, ici cité par Jouin (op. cit. note 3 [1878], I, p. 479, note 1), ajoutait : « il raccommodera leur statue, s'en ira enchanté et croira encore leur devoir du retour ».

71. H. Jouin, op. cit. note 3 [1878], I, pp. 479-480.

72. A. Bruel, op. cit. note 18, II, pp. 409-410.

73. Id., p. 411.

74. Il l'évoque dans une lettre à Victor Pavie, le 10 janvier 1853 ; voir H. Jouin, op. cit. note 6 [1890], p. 309. L'information figure à nouveau dans le Journal des Arts, le 27 juillet 1866, p. 4 («Nouvelles», reprenant un article du Moniteur des Arts du 20 juillet), au moment de l'achèvement de la restauration du marbre en France (voir infra). « Pallikares » était le nom donné aux combattants contre les Turcs pendant la guerre d'indépendance.

75. H. Jouin, op. cit. note 6 [1878], I, p. 594, « document XXXIX ».

76. Le livret du Salon de 1827 précisait, comme nous l'avons vu, que la statue était en marbre des Pyrénées. Dans une note du 8 juin 1840, publiée par H. Jouin (op. cit. note 3, [1878], II, pp. 343-344), David donne son appréciation sur ce marbre : « Deux carrières sont maintenant en pleine activité [...] : ce sont celles de Louvie et de Saint-Béat. Le marbre de Louvie est d'un grain très fin ; depuis 1829 , j'ai exécuté un très grand nombre d'ouvrages avec des blocs de cette provenance, dont la teinte était aussi belle que celle des marbres de Carrare [...]. Le marbre de Saint-Béat a le grain un peu moins fin que celui de Louvie [...]. On trouve une assez grande variété de tons dans les blocs : ceux dont la couleur tire sur une légère nuance grisâtre, avec de petits points brillants, ressemblent beaucoup au marbre grec $»$. Si on s'en tient à cette note, le marbre de La Jeune Grecque ayant été commencé avant 1829, aurait de fortes chances d'être du Saint-Béat, ce qui correspondrait plutôt, par ailleurs, aux caractéristiques données ici par David.

77. G. Geroyanis-Petimezas, op. cit. note 2, p. 186.

78. G. Geroyanis-Petimezas, op. cit. note 2, p. 187, qui publie une photographie du Général devant La Jeune Grecque.

79. Cat. d'exp., David d'Angers, 1788-1856, Paris, Hôtel de la Monnaie, juin-septembre 1966, pp. $47-48, \mathrm{n}^{\circ}$ 58. La notice indique même que « la statue en marbre connut un triomphe au Salon de $1827 » !$

80. Lettre publiée par André Bruel, « Histoire d'une statue : La Jeune Grecque au tombeau de Marco Botzaris à Missolonghi. Marbre de David d'Angers ", Bulletin de la Société historique et ethnologique de Grèce, Athènes, 1967, pp. 360-366. 


\section{RÉSUMÉS}

Mal connue en France du fait de la place secondaire de la sculpture vis-à-vis de la peinture dans la conscience culturelle du public contemporain concernant le XIX ${ }^{\mathrm{e}}$ siècle, mais aussi de la faible représentation, au Louvre (comme dans la plupart des grands musées de Beaux-Arts) des créations de David, La Jeune Grecque occupe pourtant dans la sculpture française de la première moitié du XIX ${ }^{\mathrm{e}}$ siècle une place centrale. Si l'intérêt de David pour Botzaris et la cause grecque, la façon dont il trouva son idée et son attachement à sa statue sont assez bien renseignés, une partie de l'histoire de cette œuvre est restée dans l'ombre : la date exacte de sa réalisation, la connaissance qu'on en eut en France avant son envoi en Grèce, les circonstances de cet envoi, les contacts avec les Grecs, la place de David dans le mouvement philhellène, sa relation sentimentale et phantasmatique avec la Grèce, ses projets d'y partir installer une école de sculpture, et enfin la brutalité de sa déception en 1852. Cet article apporte un éclairage nouveau sur cette œuvre dont l'intérêt est à la fois historique et artistique : non seulement parce que le destin de cette statue raconte un moment des relations entre deux pays, la France et la Grèce, mais aussi parce que cette transplantation ratée - peut-être impossible - d'un artiste déjà consacré est révélatrice du cheminement culturel difficile, long et douloureux d'une jeune nation dans le concert européen de l'époque.

Little-known in France because of the secondary place of sculpture vis-à-vis painting in the cultural consciousness of the contemporary public concerning the nineteenth century, as well as because of the poor representation in the Louvre (as in most of the great fine art museums) of the works of David d'Angers, Reviving Greece nevertheless occupies a central place in French sculpture of the first half of the nineteenth century. While David d'Angers' interest in Botzaris and the Greek cause, and the way he came up with his idea and his attachment to his statue are fairly well-documented, part of the history of this work has remained in the dark: the exact date of its execution, the knowledge of it in France before it was sent to Greece, the circumstances of its sending, the contacts with the Greeks, David's place in the philhellenic movement, his sentimental and phantasmatic relationship with Greece, his plans to leave and set up a school of sculpture there, and finally the brutality of his disappointment in 1852. This article sheds new light on this work, which is of both historical and artistic interest not only because the fate of the statue recounts a moment in the relations between two countries, France and Greece, but also because of the failed - and perhaps impossible - transplantation of an already-established artist reveals the difficult, long and painful cultural journey of a young nation in the European concert of the time.

\section{INDEX}

Keywords : David d'Angers, nineteenth century, sculpture, Botzaris, philhellenic movement, Greece, exile, Republican exile, Romanticism, tomb, war of independence

Index chronologique : XIXe siècle

Mots-clés : David d'Angers, Sculpture, Botzaris, mouvement philhellene, Grèce, Exil, proscrit républicain, Romantisme, Tombeau, Guerre d'indépendance 


\section{AUTEUR}

\section{PHILIPPE DUREY}

Conservateur général honoraire du Patrimoine, Philippe Durey est ancien élève de l'École du Louvre et diplômé de Sciences politiques. Il a successivement été conservateur à l'Inspection générale des musées, directeur du musée des Beaux-Arts de Lyon, administrateur général de la Réunion des Musées Nationaux et enfin, de 2002 à 2017, directeur de l'École du Louvre. Au cours d'une carrière en grande partie consacrée à l'administration et à la gestion des musées, il a également assuré le commissariat de nombreuses expositions, conduit d'importants travaux d'aménagements muséographiques, et rédigé de nombreux articles concernant son domaine de spécialité, la sculpture du XIX ${ }^{\mathrm{e}}$ siècle, qu'il a enseigné à l'École du Louvre.

Honorary general heritage curator Philippe Durey is a former student of the École du Louvre and a graduate of the Institut d'Études Politiques de Paris. He was successively curator at the Inspection Générale des Musées, director of the Musée des Beaux-Arts de Lyon, director at large of the Réunion des Musées Nationaux and finally, from 2002 to 2017, director of the École du Louvre. During a career largely devoted to the administration and management of museums, he has also curated numerous exhibitions, led major museum development projects and written numerous articles on his specialist field, nineteenth-century sculpture, which he taught at the École du Louvre. 